\title{
Comparison of Trajectory Tracking Controllers for Emergency Situations
}

\author{
Daniel Heß, Matthias Althoff, Thomas Sattel
}

\begin{abstract}
Over the last years a number of different vehicle controllers has been proposed for tracking planned paths or trajectories. Most of previously published works do not compare their results with other approaches or limit the comparison to a few scenarios. Unfortunately, comparisons with existing controller concepts are very rare and a ranking is hard to establish from the literature. In this work, we rigorously compare inversion-based trajectory tracking controllers by systematically exploring the set of possible solutions when disturbances vary over time and initial states and parameters are uncertain. By using Monte-Carlo simulation, we determine the average performance and by using rapidly exploring random trees, we determine the worst-case performance, which is especially important in emergency situations when avoiding a crash is essential. The tested scenarios and the applied methodologies are documented in detail so that they serve as benchmark problems for other control concepts. The results show that the controller with smaller relative degree performs better with respect to the worst-case deviation computed by rapidly exploring random trees, while conventional simulations of random scenarios would not reveal any difference.
\end{abstract}

\section{INTRODUCTION}

Recent advancements of safety systems in road vehicles have shown that collision avoidance will be the dominant future technology for safer vehicles. A major advantage of collision avoidance systems is that they require only a few lightweight parts, such as environment sensors and computer hardware, whereas passive safety systems significantly increase the vehicle mass while still causing injuries or fatalities.

We consider collision avoidance systems which fully take over the vehicle control in emergency situations. This is typically realized by a two-stage approach, consisting of a path/trajectory planning phase and a path/trajectory tracking phase. An alternative are reactive approaches, which do not plan ahead [13]. Due to their simplicity, reactive approaches are popular with indoor robots, but are not suitable for road vehicles since it is hard to ensure all constraints on the motion when the plan has no look-ahead horizon.

In this work, we focus on trajectory tracking and assume that an evasive trajectory is already planned as described in e.g. [10]. There exist good solutions for trajectory tracking when the vehicle is not operated at its physical limits as demonstrated by fully automated vehicles at the DARPA Urban Challenge [5]. However, designing a controller that operates the vehicle at its physical limits is an open problem.

M. Althoff is with the department of Computer Science and Automation, D. Hess and T. Sattel are with the department of Mechanical Engineering, Ilmenau University of Technology, 98693 Ilmenau, Germany. \{Daniel.Hess, Matthias.Althoff, Thomas.Sattel \}eTU-Ilmenau.de
Extreme maneuvers are typically applied when a collision is almost inevitable so that the requirements on the tracking performance in those situations are especially high since a crash is possibly no longer avoided when the deviation is too large.

Many trajectory tracking approaches apply input/output (I/O) linearization [9]. Early results [12] used linear tire models, but approaches with more realistic, nonlinear tire models are known [24]. The tracking error can be expressed in earth-fixed coordinates [21] or trajectory-fixed coordinates [24] and may be compensated by an inversion-based nonlinear mapping of a linear feedback-controller. A subset of the input/output linearizable systems is full-state linearizable, which are known as flat systems [6], possessing the beneficial property of a possible static mapping from the desired trajectory to the full vehicle state and input vector [8]. Whether this property may be achieved depends on the vehicle modeling assumptions and the available control inputs. For example, individual wheel steering [14], differential breaking of leftand right wheels [1] as well as differential breaking of front and rear wheels [17] may lead to flat systems. Alternatively, the system description may be simplified, e.g., neglecting the longitudinal dynamics [2], [23] or focusing only on velocities and neglecting the vehicle position [7]. It is also known that the zero side-slip assumption of a kinematic vehicle model facilitates flatness [20]. Alternative approaches for path/trajectory tracking are Lyapunov-based control designs [3], [19] and sliding mode controllers [18].

All previously mentioned works in the literature have in common that they have never been evaluated using a set of standardized tests. To the best knowledge of the authors no such benchmark tests have been published for tracking controllers of vehicles. Benchmark problems have accelerated research in many areas, such as artificial intelligence (RoboCup Federation), SAT-Solvers (SATLIB library), computer-vison (e.g. KITTI Vision Benchmark Suite), and powers systems (IEEE benchmark problems), to name only a few.

The contribution of this work is twofold. First, we propose a benchmark for path/trajectory tracking controllers of road vehicles. Besides the benchmark itself, we propose several performance measures that quickly highlight the strengths and weaknesses of the controller. Among them is a worstcase deviation obtained by rapidly exploring random trees (RRTs), a mean deviation obtained by Monte-Carlo simulation, and a parameter sensitivity analysis. The second contribution is a comparison of inversion-based controllers based on the work in [24] and [7] using the proposed 
benchmarks. All details of the benchmark problems are fully available for download ${ }^{1}$. Based on future feedback, we plan to continuously improve and include new proposed benchmarks. Besides the description of the benchmarks, we also provide the MATLAB code which makes it possible to simulate all the benchmark problems for any type of controller, enabling other researchers to compare their results with the ones published in this work.

The paper is organized as follows. In Sec. II, we present the vehicle model used for the comparison. Based on this model, we derive the controllers from [24] and [7] for the comparison. This is done in a two-stage process: First, the controllers for tracking a desired velocity vector are derived in Sec. III, which are used in Sec. IV to derive the controllers for position tracking. In Sec. V, the framework for comparing both controllers is introduced, which is suitable for comparing other controllers, too. The results of the comparison are discussed in Sec. VI, followed by the conclusion in Sec. VII.

\section{VEHICLE MODEL}

We investigate the vehicle trajectory tracking behavior without differential breaking, therefore a bicycle model (see Fig. 1) is sufficient to describe the dynamics. The task is to achieve that the look-ahead point $P$ tracks a trajectory $\tau(t)=\left[X^{D}(t), Y^{D}(t)\right]^{T}$ of desired $\mathrm{x}$ - and y-positions $X^{D}, Y^{D}$ (see Fig. 1). The tangential velocity of $\tau(t)$ is $v(t)$, the orientation of the velocity vector is $\theta(t)$.

The bicycle model combines wheels of the front and rear axle, where $l_{f}$ and $l_{r}$ are the distances to the the center of gravity $C G$. We denote the front wheel steering angle by $\delta$, the rotational velocity by $\omega$, and the angle of the vehiclefixed coordinate system relative to the earth-fixed coordinate system $\left[e_{X}, e_{Y}\right]$ by $\psi$. The velocity vectors at the center of gravity and the look-ahead point $\mathrm{P}$ are given in vehicle-fixed coordinates. Using the rotation matrix $R(\cdot)=\left(\begin{array}{cc}\cos (\cdot) & -\sin (\cdot) \\ \sin (\cdot) & \cos (\cdot)\end{array}\right)$ the velocity of the point $\mathrm{P}$ in earth-fixed coordinates is $\left[\dot{X}^{P}, \dot{Y}^{P}\right]^{T}=R(\psi) v_{x y}^{P}, v_{x y}^{P}=\left[v_{x}^{P}, v_{y}^{P}\right]^{T}$.

Basically there are two common formulations to describe the planar motion of the vehicle. One can either use the absolute velocity of the center of gravity, $v^{C G}$, and the slip-angle $\beta$, or the vehicle-fixed velocity components $\left[v_{x}^{C G}, v_{y}^{C G}\right]$ used herein, where $v^{C G}=\left\|\left[v_{x}^{C G}, v_{y}^{C G}\right]\right\|$ and $\beta=$ $\arctan \left(v_{y}^{C G} / v_{x}^{C G}\right)$. We further introduce the partial state vector $x_{d}=\left[v_{x}^{C G}, v_{y}^{C G}, \omega\right]^{T}$ of velocity variables. Using the tire force

\footnotetext{
${ }^{1} \mathrm{http}: / /$ vehiclecontrol.it.cx/ttb
}

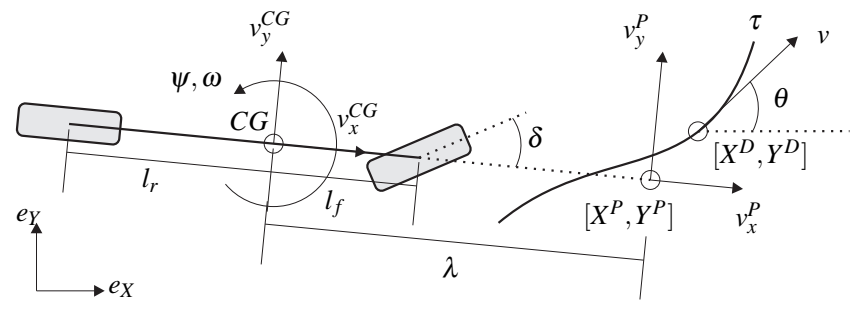

Fig. 1. Bicycle model vectors of the front and rear axle, $F_{x y, f}$ and $F_{x y, r}$ measured in the vehicle-fixed coordinate system, a moment balance yields the following function $f_{d}$, and the velocity vector of the point $P$ is selected as the output $h_{d}$ :

$$
\begin{gathered}
f_{d}:\left\{\begin{array}{c}
\dot{v}_{x}^{C G}=\left(F_{x, f}+F_{x, r}\right) / m+v_{y}^{C G} \omega \\
\dot{v}_{y}^{C G}=\left(F_{y, f}+F_{y, r}\right) / m-v_{x}^{C G} \omega \\
\dot{\omega}=\left(l_{f} F_{y, f}-l_{r} F_{y, r}\right) / J
\end{array}\right. \\
h_{d}\left(x_{d}\right)=\left(\begin{array}{c}
v_{x}^{P} \\
v_{y}^{P}
\end{array}\right)=\left(\begin{array}{c}
v_{x}^{C G} \\
v_{y}^{C G}+\lambda \omega
\end{array}\right)
\end{gathered}
$$

Since we aim at controlling the vehicle at its physical limits, a nonlinear tire model that reflects the saturation of forces at higher slip values is indispensable. Furthermore, for combined breaking and evasion, the interdependence of longitudinal, lateral and normal tire forces has to be considered. For that reason, we use a tire model proposed by Orend [14], which is explicitly invertible. Exemplary for the combined front wheel, the following quantities influence the resulting tire force: The slip vector $s_{x y}^{W F}$ is given in vehiclefixed coordinates as the normalized difference between the velocity of the vehicle body at the center of the front wheel $v_{x y}^{W F}=\left[v_{x}^{C G}, v_{y}^{C G}+l_{f} \omega\right]^{T}$ and the velocity of the tire patch relative to the vehicle body $R(\delta)\left[r \omega_{f}, 0\right]^{T}$, where $\omega_{f}$ is the rotational velocity of the wheel and $r$ the wheel radius (see (2)). We limit the analysis to the case of breaking, implying that $\left\|v_{x y}^{W F}\right\|>\left|r \omega_{f}\right|$. The scalar tire force model is similar to Pacejka's [15] approach with the constants $B$ and $C$. The vector $\mu_{x y}$ is unit-less and describes the fraction of the normal force utilized as tire force in $x$ and $y$ direction. The equations of the tire model are:

$$
\begin{aligned}
& s_{x y, f}=\left(v_{x y}^{W F}-R(\delta)\left(\begin{array}{c}
r \omega_{f} \\
0
\end{array}\right)\right) /\left\|v_{x y}^{W F}\right\| \\
& \mu_{x y}\left(s_{x y}\right)=-\frac{s_{x y}}{\left\|s_{x y}\right\|} \cdot \sin \left(C \arctan \left(B / \mu_{0} \cdot\left\|s_{x y}\right\|\right)\right) \\
& F_{x y, f}=\mu_{x y}\left(s_{x y, f}\right) \cdot \mu_{0} \cdot F_{z, f}
\end{aligned}
$$

The tire forces for the rear axle are calculated accordingly with $\delta=0$. As in [22], the tire normal force can be modeled under the assumption of an equilibrium of moments for zero pitch angle and the height $h$ of the $\mathrm{CG}$ :

$$
F_{z, f}=m g \cdot \frac{l_{r}-h \mu_{x, r}}{l_{f}+l_{r}+h\left(\mu_{x, f}-\mu_{x, r}\right)}
$$

A second part of the model concerning the positions is required to describe the trajectory tracking dynamics. The partial state vector $x_{p}=\left[X^{P}, Y^{P}, \psi\right]^{T}$ of position variables is defined with respect to the earth-fixed coordinates of $P$ and the vehicle orientation $\psi$. The output $h_{p}$ of the position level system is defined as the tracking error $\varepsilon_{t n}$ in trajectory-fixed coordinates with vector components in path tangential $t$ and path normal $n$ directions. The corresponding state equation 
$f_{p}$ uses the output of the velocity level system $h_{d}=v_{x y}^{P}$ :

$$
\begin{aligned}
& f_{p}:\left\{\begin{aligned}
\left(\begin{array}{c}
\dot{X}^{P} \\
\dot{Y}^{P}
\end{array}\right) & =R(\psi) v_{x y}^{P} \\
\dot{\psi} & =\omega
\end{aligned}\right. \\
& h_{p}\left(x_{p}, t\right)=\varepsilon_{t n}=R(-\theta(t))\left[\left(\begin{array}{c}
X^{P} \\
Y^{P}
\end{array}\right)-\left(\begin{array}{l}
X^{D}(t) \\
Y^{D}(t)
\end{array}\right)\right]
\end{aligned}
$$

It is well-known from the literature that (1) and (4) are input/output linearizable, i.e., one can transform the system such that the input-output behavior from input vector $\left[\delta, \omega_{f}\right]$ to output vector $h_{p}$ is linear. Depending on the choice of the look-ahead distance $\lambda$, one obtains input/output linearizations of different relative degree ${ }^{2}$ with respect to the output $h_{d}$. In order to describe the input/output linearization in two steps, we first obtain such a linearization for (1) in Sec. III and then for (4) in Sec. IV.

\section{VELOCITY CONTROL}

In this section, the input/output linearization is performed for (1), which is later used for the position control of the vehicle. As the tire model (2) is invertible up to the maximum tire force, the front axle tire forces can be defined as a virtual input for (1):

$$
u_{d}=\left[F_{x, f}, F_{y, f}\right]^{T}
$$

If the tire force exceeds the maximum force, the absolute value of $u_{d}$ is limited to its maximum.

Independently from the choice of the look-ahead distance $\lambda$ (Fig. 1), the output $h_{d, 1}$ has a relative degree of one. This can be shown by inserting the right-hand side of (1) into $h_{d, 1}^{(1)}:=\frac{\mathrm{d} h_{d, 1}}{\mathrm{~d} t}=\dot{v}_{x}^{C G}$, which yields after reordering:

$$
u_{d, 1}=F_{x, f}=m\left(h_{d, 1}^{(1)}-v_{y}^{C G} \omega\right)-F_{x, r}
$$

\section{A. Front decoupling point}

Choosing $\lambda=J /\left(l_{r} m\right)$, which we refer to as the front decoupling point, the output $h_{d, 2}$ also has a relative degree of one. Analogously to obtaining (6), we have

$$
\begin{gathered}
h_{d, 2}^{(1)}=\frac{l_{f}+l_{r}}{l_{r} m} F_{y, f}-v_{x}^{C G} \omega \\
\Rightarrow u_{d, 2}=F_{y, f}=\frac{l_{r} m}{l_{f}+l_{r}}\left(h_{d, 2}^{(1)}+v_{x}^{C G} \omega\right)
\end{gathered}
$$

For the front decoupling point, the change of lateral velocity is not influenced by the rear tire force, which could be useful when the rear tire forces are uncertain.

In many aspects similar to the front decoupling point are all choices of $\lambda>-J /\left(l_{f} m\right)$ :

$$
h_{d, 2}^{(1)}=\frac{J+\lambda l_{f} m}{m J} F_{y, f}-\frac{J-\lambda l_{r} m}{m J} F_{y, r}-v_{x}^{C G} \omega
$$

Here, an influence of the rear tire forces remains in $h_{d, 2}^{(1)}$, but if known, it can be easily compensated. The following

\footnotetext{
${ }^{2}$ The relative degree corresponds to the number of output derivatives necessary until the corresponding input variable appears.
}

control input can be used to acquire a desired change in lateral velocity of the reference position $h_{d, 2}^{(1)}$, which is a generalized version of (8):

$$
u_{d, 2}=F_{y, f}=\frac{m J}{J+\lambda l_{f} m}\left(h_{d, 2}^{(1)}+\frac{J-\lambda l_{r} m}{m J} F_{y, r}+v_{x}^{C G} \omega\right)
$$

Similar control concepts are applied in [16], [24].

\section{B. Rear decoupling point}

Similar to the independence of the front decouplingpoint acceleration from the rear wheel forces, a position on the vehicle $\mathrm{x}$-axis must exist, where the change in lateral velocity is independent from the front wheel force. In [7] this knowledge is applied to show the flatness of a nonlinear bicycle system equivalent to (1). Choosing $\lambda=-J /\left(l_{f} m\right)$, the output $h_{2}$ has a relative degree of two, if one assumes the rear lateral tire force to be independent from all inputs. For the applied tire model though, this assumption stands in conflict with the following two properties: a) the rear tire lateral force $F_{y, r}$ depends on the rear normal force $F_{z, r}$ which is in turn influenced by $u_{d, 1}=F_{x, f}$ and b) the tire force $F_{y, r}$ decreases for large longitudinal slip values. We therefore have to increase the relative degree of $h_{d, 1}$ by one integration step, lifting $h_{d, 1}^{(1)}$ into the state vector and now directly controlling the output-function derivatives $h_{d, 1}^{(2)}$ and $h_{d, 2}^{(2)}$. The corresponding equations for the lateral velocity output are:

$$
\begin{aligned}
h_{d, 2}^{(1)}= & \frac{l_{f}+l_{r}}{l_{f} m} F_{y, r}\left(v_{x}^{C G}, v_{y}^{C G}, \omega, h_{d, 1}^{(1)}\right)-v_{x}^{C G} \omega \\
h_{d, 2}^{(2)}= & \frac{l_{f}+l_{r}}{l_{f} m} \frac{\partial F_{y, r}}{\partial\left[x_{d}, h_{d, 1}^{(1)}\right]^{T}}\left[f_{d}\left(x_{d}, u_{d, 1}, u_{d, 2}\right), h_{d, 1}^{(2)}\right]^{T} \\
& -h_{d, 1}^{(1)} \omega-v_{x}^{C G} \dot{\omega}
\end{aligned}
$$

The front lateral tire force $u_{d, 2}$ is determined by solving (12).

\section{POSITION CONTROL}

The ultimate goal for applications such as collision avoidance is to control the position of the vehicle. From the position control derived in this section, one obtains the required changes in lateral and longitudinal velocity $\left(h_{d, 1}^{(1)}, h_{d, 2}^{(1)}\right)$ for the front decoupling point, or $\left(h_{d, 1}^{(1)}, h_{d, 2}^{(2)}\right)$ for the rear decoupling point. These desired values are then used to obtain the tire forces at the front wheel according to the input/output linearization of the previous section.

The deviation of the reference position from the desired trajectory can be described with the help of the outputvector $h_{d}$ of the velocity layer. By replacing the earth fixed velocity representations in the derivative of the position error with path- and vehicle-fixed representations, $\left[\dot{X}^{D}, \dot{Y}^{D}\right]^{T}=$ $R(\theta)[v, 0]^{T},\left[\dot{X}^{P}, \dot{Y}^{P}\right]^{T}=R(\psi) h_{d}$, we obtain:

$$
h_{p}^{(1)}=-\dot{\theta}\left(\begin{array}{c}
-h_{p, 2} \\
h_{p, 1}
\end{array}\right)-\left(\begin{array}{l}
v \\
0
\end{array}\right)+R(\psi-\theta) h_{d}
$$


According to (13), $h_{p}$ could be I/O-linearized with a hypothetical input $u_{p}=h_{d}$ and a relative degree of one. We can therefore continue the derivation until $h_{p}^{(r+1)}$, where $r$ is the relative degree of $h_{d}$, to achieve an I/O-linearization of $h_{p}$ with the input $u_{p}:=u_{d}$ (front tire forces).

For the front decoupling point or all $\lambda>-J /\left(l_{f} m\right)$ described in Sec. III-A, $h_{d}$ has a relative degree of one. By solving the second derivative of the position error $h_{p}^{(2)}$ for the derivative $h_{d}^{(1)}$ of the velocity of the reference position, one receives:

$$
\begin{aligned}
h_{d}^{(1)}= & R(\theta-\psi)\left(h_{p}^{(2)}+\ddot{\theta}\left(\begin{array}{c}
-h_{p, 2} \\
h_{p, 1}
\end{array}\right)+\dot{\theta}\left(\begin{array}{c}
-h_{p, 2}^{(1)} \\
h_{p, 1}^{(1)}
\end{array}\right)+\left(\begin{array}{c}
\dot{v} \\
0
\end{array}\right)\right) \\
& -(\omega-\dot{\theta})\left(\begin{array}{c}
-h_{d, 2} \\
h_{d, 1}
\end{array}\right)
\end{aligned}
$$

In case of the rear decoupling point $\lambda=-J /\left(l_{f} m\right)$ in Sec. III-B the relative degree of two requires solving $h_{p}^{(3)}$ (third derivative of the position error) for $h_{d}^{(2)}$ :

$$
\begin{aligned}
h_{d}^{(2)}= & R(\theta-\psi)\left(h_{p}^{(3)}+\dddot{\theta}\left(\begin{array}{c}
-h_{p, 2} \\
h_{p, 1}
\end{array}\right)+2 \ddot{\theta}\left(\begin{array}{c}
-h_{p, 2}^{(1)} \\
h_{p, 1}^{(1)}
\end{array}\right)\right. \\
& \left.+\dot{\theta}\left(\begin{array}{c}
-h_{p, 2}^{(2)} \\
h_{p, 1}^{(2)}
\end{array}\right)+\left(\begin{array}{c}
\ddot{v} \\
0
\end{array}\right)\right)-(\dot{\omega}-\ddot{\theta})\left(\begin{array}{c}
-h_{d, 2} \\
h_{d, 1}
\end{array}\right) \\
& -2(\omega-\dot{\theta})\left(\begin{array}{c}
-h_{d, 2}^{(1)} \\
h_{d, 1}^{(1)}
\end{array}\right)+(\omega-\dot{\theta})^{2} h_{d}
\end{aligned}
$$

Both cases constitute an input/output linearization. Accordingly a linear feedback for regulation of output $h_{p}$ can be applied. The linear feedback gains listed in Tab. II are chosen in such a way that the second- and third-order linear systems have a similar initial condition response.

\section{A. Setup of Controller A}

Both controllers assume the variables $X^{C G}, Y^{C G}, \psi, v_{x}^{C G}$, $v_{y}^{C G}$ and $\omega$ to be measured. Controller A proceeds to calculate the physical inputs $\delta$ and $\omega_{f}$ as:

1) Calculate tracking errors $h_{p}$ and $h_{p}^{(1)}$

2) Linear feedback $h_{p}^{(2)}:=-K_{A 1} h_{p}^{(1)}-K_{A 0} h_{p}$

3) Compute the required velocity change $h_{d}^{(1)}$ according to $(14)$

4) Compute the required forces according to (6) and (8)

5) Compute $\delta$ and $\omega_{f}$ by solving (2)

\section{B. Setup of Controller B}

Controller B uses the internal state $h_{d, 1}^{(1)}$ :

1) Calculate tracking errors $h_{p}, h_{p}^{(1)}$ and $h_{p}^{(2)}$

2) Linear feedback $h_{p}^{(3)}:=-K_{B 2} h_{p}^{(2)}-K_{B 1} h_{p}^{(1)}-K_{B 0} h_{p}$

3) Compute the required change $h_{d}^{(2)}$ according to (15)

4) Integrate $h_{d, 1}^{(1)}=\int h_{d, 1}^{(2)} \mathrm{d} t$
5) Compute the required forces according to (6) and (12)

6) Compute $\delta$ and $\omega_{f}$ by solving (2)

\section{FRAMEWORK FOR COMPARISON}

In this section, we provide detailed information for two typical emergency maneuvers, which can serve as benchmark problems for other trajectory tracking control concepts. The first scenario is a lane change under braking and the second one is a double lane change under braking. We plan to add new scenarios to our website as soon as we discover that other scenarios reveal weaknesses that are not observed by the current scenarios.

\section{A. Trajectories}

The parameters for the nominal trajectories for both scenarios are provided in Tab. I, where all values are with respect to the center of gravity. The road-fixed Cartesian coordinates $Y$ and $X$ are chosen as polynomials of an auxiliary variable $r$ and the function $S$ as a polynomial which maps the time to a distance along the path $[X(r), Y(r)]$. The degree of the polynomials $X, Y$ and $S$ are chosen to fulfill the given boundary conditions, so that the functions are uniquely defined by them. The arc length $L(r)=\int_{0}^{r} \sqrt{\left[X^{\prime}\right]^{2}(\rho)+\left[Y^{\prime}\right]^{2}(\rho)} \mathrm{d} \rho$ of the path is com-

\begin{tabular}{|c|c|c|c|c|c|c|}
\hline \multicolumn{5}{|c|}{ SCENARIO I - LANE CHANGE } & \multirow[b]{2}{*}{$Y^{\prime}(40 m)$} & \multirow[b]{2}{*}{$Y^{\prime \prime}(40 m)$} \\
\hline$Y(0 m)$ & $Y^{\prime}(0 m)$ & $Y^{\prime \prime}(0 m)$ & I & $Y(40 m)$ & & \\
\hline $0 \mathrm{~m}$ & $0 \mathrm{~m} / \mathrm{s}$ & $0 \mathrm{~m} / \mathrm{s}^{2}$ & I & $3 \mathrm{~m}$ & $0 \mathrm{~m} / \mathrm{s}$ & $0 \mathrm{~m} / \mathrm{s}^{2}$ \\
\hline$S(0 s)$ & $S^{\prime}(0 s)$ & $S^{\prime \prime}(0 s)$ & I & $S(2 s)$ & I & $S^{\prime \prime}(2 s)$ \\
\hline $0 \mathrm{~m}$ & $22 \mathrm{~m} / \mathrm{s}$ & $0 \mathrm{~m} / \mathrm{s}^{2}$ & I & $40.2 \mathrm{~m}$ & I & $0 \mathrm{~m} / \mathrm{s}^{2}$ \\
\hline \multicolumn{5}{|c|}{ SCENARIO II - DOUBLE LANE CHANGE } & & \\
\hline$Y(0 m)$ & $Y^{\prime}(0 m)$ & $Y^{\prime \prime}(0 m)$ & $\mathrm{Y}(35 \mathrm{~m})$ & $Y(70 m)$ & $Y^{\prime}(70 m)$ & $Y^{\prime \prime}(70 m)$ \\
\hline $0 \mathrm{~m}$ & $0 \mathrm{~m} / \mathrm{s}$ & $0 \mathrm{~m} / \mathrm{s}^{2}$ & $3 \mathrm{~m}$ & $-1 \mathrm{~m}$ & $0 \mathrm{~m} / \mathrm{s}$ & $0 \mathrm{~m} / \mathrm{s}^{2}$ \\
\hline$S(0 s)$ & $S^{\prime}(0 s)$ & $S^{\prime \prime}(0 s)$ & I & $S(4 s)$ & I & $S^{\prime \prime}(4 s)$ \\
\hline $0 \mathrm{~m}$ & $22 \mathrm{~m} / \mathrm{s}$ & $0 \mathrm{~m} / \mathrm{s}^{2}$ & I & $70.5 \mathrm{~m}$ & I & $0 \mathrm{~m} / \mathrm{s}^{2}$ \\
\hline
\end{tabular}
puted numerically to define the trajectory as $[X(t), Y(t)]:=$ $[X(r(t)), Y(r(t))]$ with $r(t)=L^{-1}(S(t))$

TABLE I

SCENARIO PARAMETERS

In order to track the given trajectories $[X(t), Y(t)]$ with respect to the center of gravity for both controllers, we derive the corresponding trajectories for the reference position $\left[X^{D}(t), Y^{D}(t)\right]$ such that the center of gravity is moved along $[X(t), Y(t)]$ in case of error free tracking (see [24]). As a first step, the initial value problem for the zero dynamic states is numerically solved with $\lambda=0$ and for the path $[X(t), Y(t)]$ to acquire $\psi_{z}(t)$ and $\omega_{z}(t)$. By applying the input $u_{d, 2}$ from (10) for the open-loop control, one obtains:

$$
\begin{aligned}
J \dot{\omega} & =l_{f} m a_{y}-\left(l_{f}+l_{r}\right) F_{y, r} \\
\text { with } \quad a_{y} & =(\dot{\theta}-2 \omega) v \cos (\theta-\psi)+\dot{v} \sin (\theta-\psi)
\end{aligned}
$$

Then the trajectory positions can be translated by $\lambda$ to the instantaneous yaw direction of the vehicle:

$$
\left(\begin{array}{l}
X^{D}(t) \\
Y^{D}(t)
\end{array}\right)=R\left(\psi_{z}(t)\right)\left(\begin{array}{l}
\lambda \\
0
\end{array}\right)+\left(\begin{array}{l}
X(t) \\
Y(t)
\end{array}\right) .
$$


TABLE II

MODEL PARAMETERS

\begin{tabular}{|c|c|c|c|c|c|}
\hline \multicolumn{3}{|c|}{ VEHICLE (from [14]) } & & \multirow[b]{2}{*}{$h(\mathrm{~m})$} & \\
\hline$m(\mathrm{~kg})$ & $J\left(\mathrm{~kg} \mathrm{~m}^{2}\right)$ & $l_{f}(\mathrm{~m})$ & $l_{r}(\mathrm{~m})$ & & \\
\hline 1750 & 2500 & 1.43 & 1.27 & 0.5 & \\
\hline$r(\mathrm{~m})$ & $B_{f}$ & $C_{f}$ & $B_{r}$ & $C_{r}$ & \\
\hline 0.32 & 10.4 & 1.3 & 21.4 & 1.1 & \\
\hline \multicolumn{3}{|c|}{ CONTROLLER } & & & \\
\hline$K_{A 1}$ & $K_{A 0}$ & $K_{B 2}$ & $K_{B 1}$ & $K_{B 0}$ & \\
\hline 3.35 & 5 & 5.87 & 17.3 & 22.4 & \\
\hline \multicolumn{3}{|c|}{ MEASUREMENT ERRORS } & & & \\
\hline$\hat{\varepsilon}_{X C G}(\mathrm{~m})$ & $\hat{\varepsilon}_{Y C G}(\mathrm{~m})$ & $\hat{\varepsilon}_{\psi}\left({ }^{\circ}\right)$ & $\hat{\varepsilon}_{v_{X}^{C G}}(\mathrm{~m} / \mathrm{s})$ & $\hat{\varepsilon}_{v_{V}^{C G}}(\mathrm{~m} / \mathrm{s})$ & $\hat{\varepsilon}_{\omega}(\% / \mathrm{s})$ \\
\hline 0.05 & 0.05 & 1 & 0.05 & 0.05 & 1 \\
\hline
\end{tabular}

\section{B. Test Methodology}

In the nominal case, when there is no initial deviation from the trajectory, no measurement noise, known parameters and unsaturated wheel forces, the controllers exactly track the desired trajectory in open-loop mode due to the inversionbased approach. In reality none of the above assumptions are precisely met, resulting in different trajectories and control inputs for the two controllers. We use the following measures to compare the tracking performance according to the deviation $\varepsilon^{C G}(t)=R(-\theta)\left[X^{C G}(t)-X(t), Y^{C G}(t)-Y(t)\right]^{T}$ of the $\mathrm{CG}$ :

$$
\begin{aligned}
\text { maximum deviation: } & \max _{t \in[0, T]}\left|\varepsilon_{\{t, n\}}^{C G}(t)\right| \\
\text { average deviation: } & \frac{1}{T} \int_{0}^{T}\left|\varepsilon_{\{t, n\}}^{C G}(t)\right| \mathrm{d} t \\
\text { final deviation: } & \varepsilon_{\{t, n\}}^{C G}(T) \\
\text { average tire saturation: } & \frac{1}{T} \int_{0}^{T}\left\|\mu_{x y\{f, r\}}(t)\right\| \mathrm{d} t
\end{aligned}
$$

With $\{t, n\}$ referring to either the tangential $(t)$ or the normal components $(n)$ and $\{f, r\}$ to the front $(f)$ and rear $(r)$ components. The following analysis methods are applied to the benchmark problems:

1) Selected Test Cases: In order to test certain properties by a few selected scenarios, we define test cases for (i) initial deviations, (ii) varying degrees of tire force saturation, and (iii) vehicle parameter variations. In test (i), the initial condition response is tested for a heading error of $\theta(0)-$ $\psi(0)=3^{\circ}$ and a lateral offset of $Y(0)-Y^{C G}(0)=0.2 \mathrm{~m}$. Tire force saturation is tested in (ii) for both trajectories using a low friction value of $\mu_{0}=0.6$ instead of $\mu_{0}=1$, which is known to the controller in a first test and unknown in a second one for which the controller assumes $\mu_{0}=1$. Besides the road friction parameters, vehicle parameters affected by the loading are investigated in test (iii). The changed parameters are $m_{e}=1.3 \cdot m, J_{e}=1.3 \cdot J$ and $l_{f, e}=1.3 \cdot l_{f}$ while the controllers are operated with the original values.

2) Monte-Carlo Simulation for Measurement Noise: The average performance under measurement errors is modeled by white Gaussian noise added to the state vector supplied to the controller. The vector $\left[X^{C G}, Y^{C G}, \psi, x_{d}\right]^{T}$ is 'measured' and the variance of each dimension of the error process is selected as $\sigma_{i}=\hat{\varepsilon}_{i}$ with the values for $\hat{\varepsilon}_{i}$ given in table II. A total number of 500 simulations is evaluated.

3) Worst-Case-Disturbance using RRTs: RRTs have been developed for planning problems in robotics [11], but are used more and more for general state space exploration [4]. We consider the problem of estimating the trajectory tracking worst case performance under noisy vehicle state measurements. The RRT algorithm is used to explore the vehicle state space in order to determine a sequence of measurement errors that maximizes the distance to the reference trajectory. Although we use the same basic technique as in [4], we make a modification to the algorithm to generate a constant number of samples for each time interval, see Fig. 2. For a point of time $t_{k+1}$ the following steps are performed:

1) Initialize the discrete set of reachable states as $\mathscr{X}\left(t_{k+1}\right)=\emptyset$.

2) Generate a random $x_{\text {sample }}$ from a multidimensional rectangle centered at $\tau\left(t_{k+1}\right)$.

3) Find the nearest state $x_{\text {near }}$ according to a distance measure $\rho$ so that $x_{\text {near }}=\arg \min \left(\rho\left(x_{\text {sample }}, x^{(i)}\right)\right)$, where $x^{(i)} \in \mathscr{X}\left(t_{k}\right)$.

4) Obtain the measurement error $\xi_{M}^{*}$ which drives $x_{\text {near }}$ to the new state $x_{\text {new }}$ closest to $x_{\text {sample: }}$ :

$$
\begin{aligned}
x^{(j)}\left(\xi_{M}\right) & =x_{\text {near }}+\int_{t_{k}}^{t_{k+1}} f\left(x(t), c\left(x(t)+\xi_{M}\right)\right) \mathrm{d} t \\
\xi_{M}^{*} & =\arg \min \left(\rho\left(x_{\text {sample }}, x^{(j)}\left(\xi_{M}\right)\right)\right) \\
x_{\text {new }} & =x^{(j)}\left(\xi_{M}^{*}\right)
\end{aligned}
$$

where $u=c(x)$ is the controller and $f=\left[f_{p}, f_{d}\right]^{T}$.

5) Add $x_{\text {new }}$ to the set of states for the next time interval $\mathscr{X}\left(t_{k+1}\right)$.

6) Repeat steps 2-5 for a predefined number of samples. When initializing $\mathscr{X}\left(t_{k+1}\right)=\mathscr{X}\left(t_{k}\right)$, one obtains an approach similar to [4]. The distance measure is chosen as

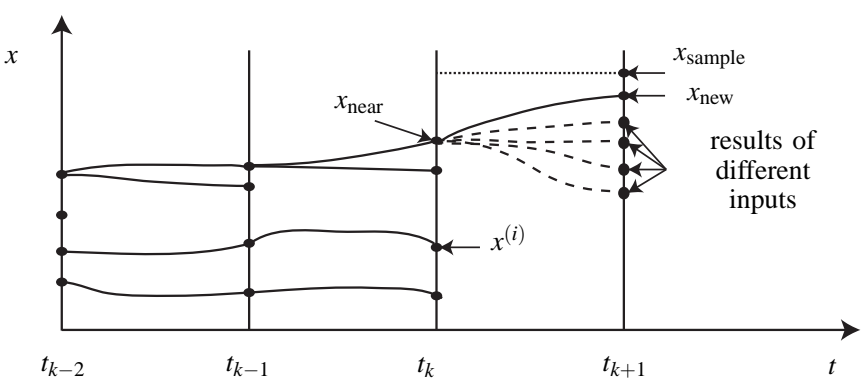

Fig. 2. RRT concept for trajectory tracking.

$\rho\left(x_{\text {sample }}, x^{(i)}\right)=\left\|N\left(x_{\text {sample }}-x^{(i)}\right)\right\|_{2}$ with a diagonal normalization matrix with values $N_{i, i}=\frac{1}{\hat{\varepsilon}_{i}}$. The measurement error $\xi_{M}^{*}$ that minimizes the distance between $x_{\text {new }}$ and $x_{\text {sample, }}$, i.e. minimizes $\rho\left(x_{a d d}, x_{s}\right)$, is chosen by testing all vertices of the set of possible measurement errors $\mathscr{X}_{M}$. The set is defined as a multidimensional rectangle with the edge lengths $\hat{\varepsilon}_{i}$ given in Tab. II. Thus, we obtain a manageable set of 64 different measurement inputs. 


\section{RESULTS}

In this section we apply the selected test cases, Monte Carlo simulations, and RRT computations from Sec. V-B to controller A and B. First, the results of the selected test cases (i-iii) summarized in Tab. III are discussed. Test (i) for investigating the effects of initial deviation shows that both controllers are quickly converging to the reference trajectory and precisely track it once the deviation is small, as shown in Fig. 3 and Fig. 4 for each scenario. The only differences are the slip angle $\beta$ and that controller B exhibits front tire force saturation in scenario II and undesirable oscillations for both scenarios. Results of test (ii) for a known, but low friction coefficient $\left(\mu_{0}=0.6\right)$ show that both controllers behave similarly in scenario I and are able to closely track the reference trajectory, but controller B is significantly worse compared to controller A in scenario II. This is because controller $B$ requires larger tire forces and thus operates longer at its physical limit. When the friction coefficient is unknown (see Sec. V-B), the average tire saturation is slightly lower and the errors are slightly higher because both controllers exceed the optimal slip value and cannot make use of the maximum tire force. Varied parameter values $m_{e}$, $J_{e}$ and $l_{f, e}$ in test (iii) lead to a static tangential offset in path tangential direction. In the comparison of path normal errors, controller A performs slightly better than controller B.

Since selected test cases only provide a snapshot of the

$\square$ nominal $+\mathrm{A} \times \mathrm{B}$
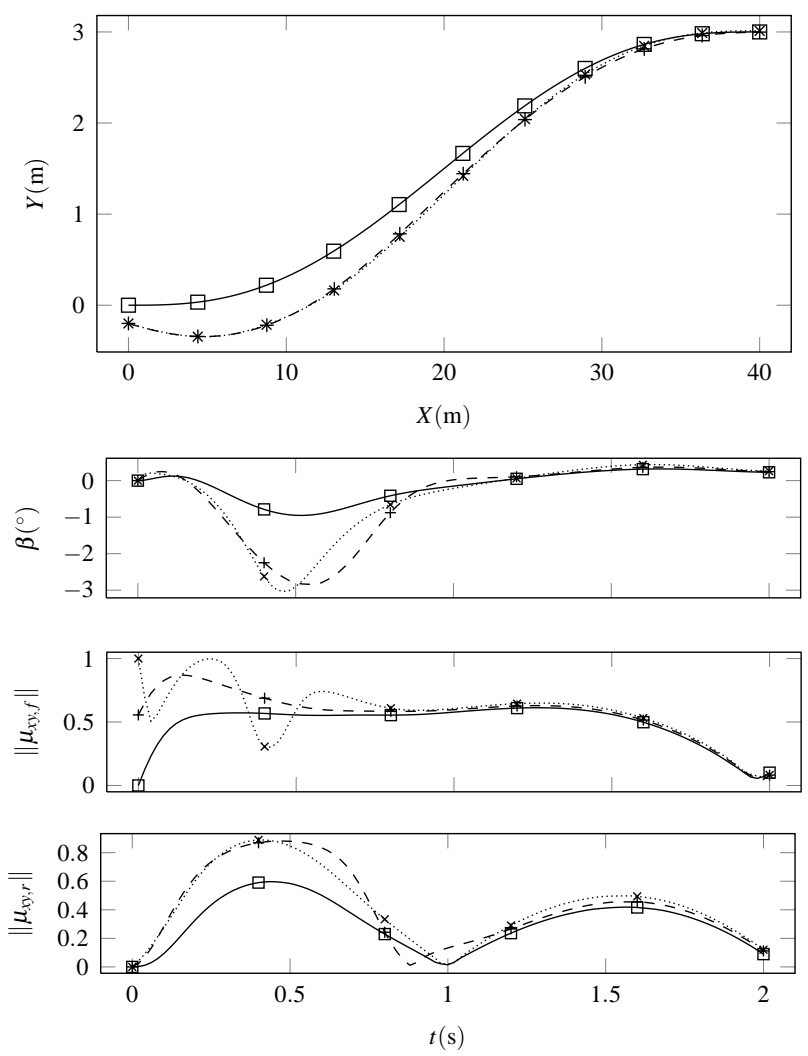

Fig. 3. Test 1 - Initial Disturbance - Scenario I controller performance, we obtained the average control performance due to sensor noise using Monte Carlo simulation as described in Sec. V-B. The sampled simulation results of the Monte-Carlo simulation are presented in Fig. 5. The average performance for both controllers is similar as presented by the standard deviation plots of the position error in Fig. 6.

Unlike the average performance, the difference in the worst-case performance of both controllers with respect to measurement noise is significant. Controller A has much lower worst-case position deviation compared to controller $\mathrm{B}$ as shown in Fig. 7. The simulation traces of the RRT computation are plotted in Fig. 8, which find larger deviations with an equal number of simulations, compared to the Monte-Carlo approach in Fig. 5. For both tests, 500 samples per time step have been used.

In summary, controller A performs slightly better or almost equal in all tests compared to controller B and is significantly better with respect to the maximum position deviation, which is especially important for evasive maneuvers, which require small deviation errors to guarantee collision avoidance. Across all tests, controller A is not so hard on the tires, i.e. tire saturation is avoided more often. The better use of tire forces is especially obvious in test (ii) for scenario II as shown in Tab. III. Tests (i-iii) showed that both controllers are insensitive to initial deviations and uncertain vehicle parameters. Using a set of test methodologies also showed
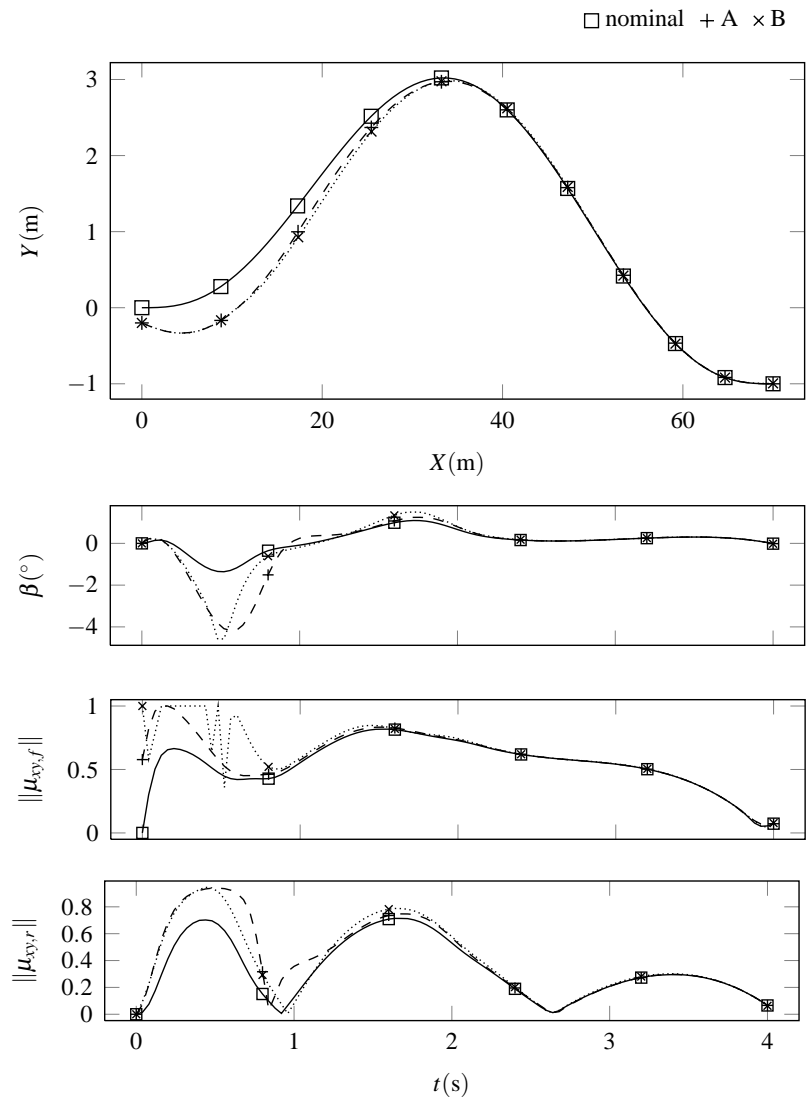

Fig. 4. Test 1 - Initial Disturbance - Scenario II 


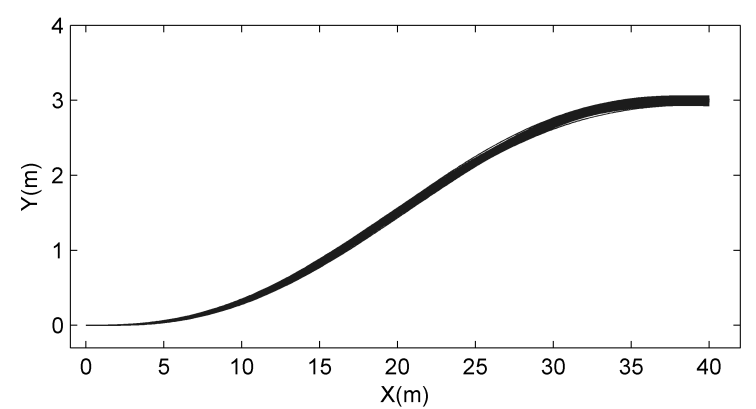

(a) Controller $\mathrm{A}$

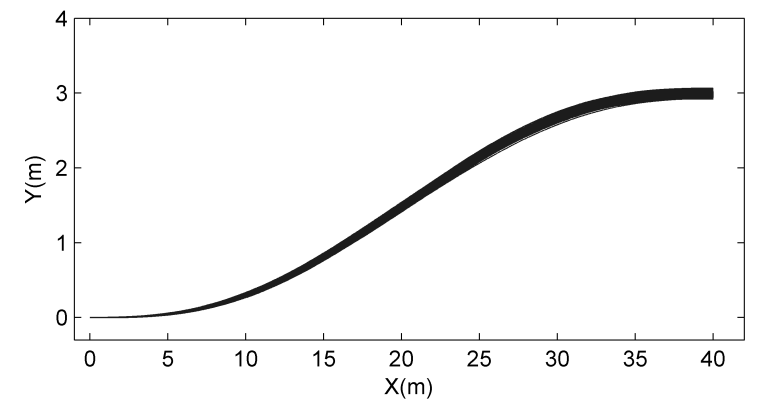

(b) Controller B

Fig. 5. Monte-Carlo Analysis
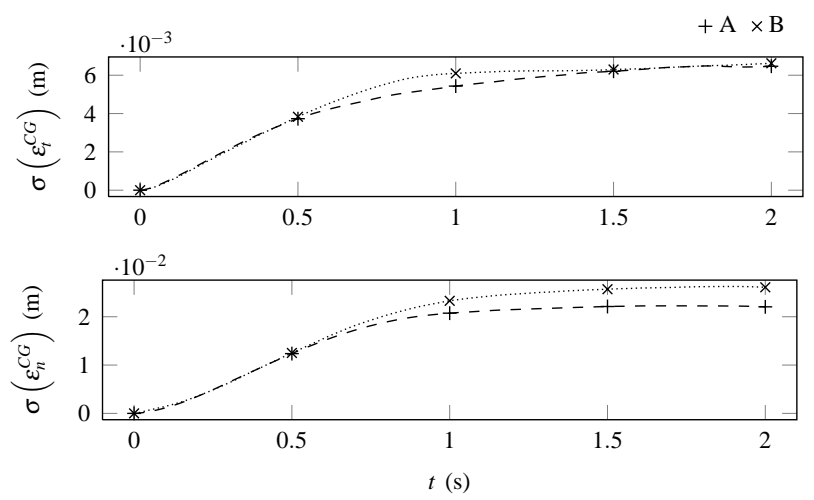

Fig. 6. Standard Deviation using Monte-Carlo Simulation
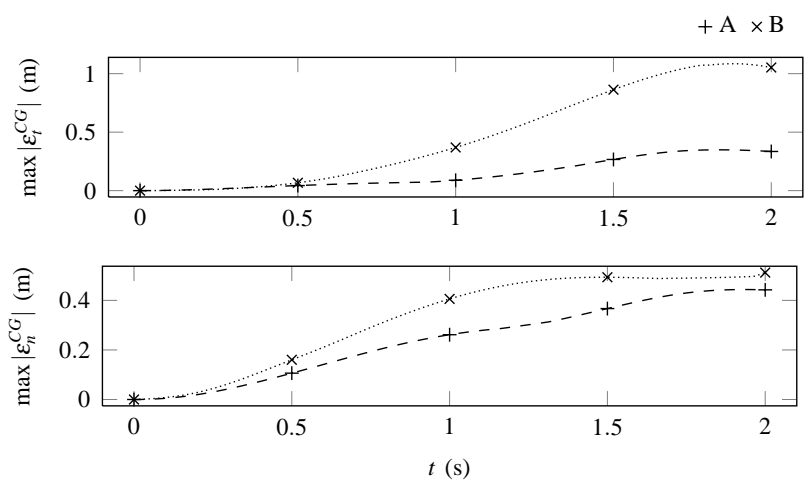

Fig. 7. Worst Case Disturbance Estimate using RRTs

that the superior performance of controller A with respect to maximum deviation errors would have been undetected without the use of RRTs.

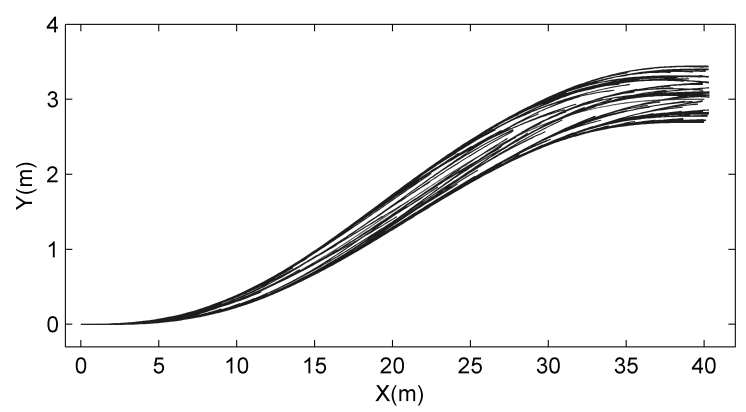

(a) Controller $\mathrm{A}$

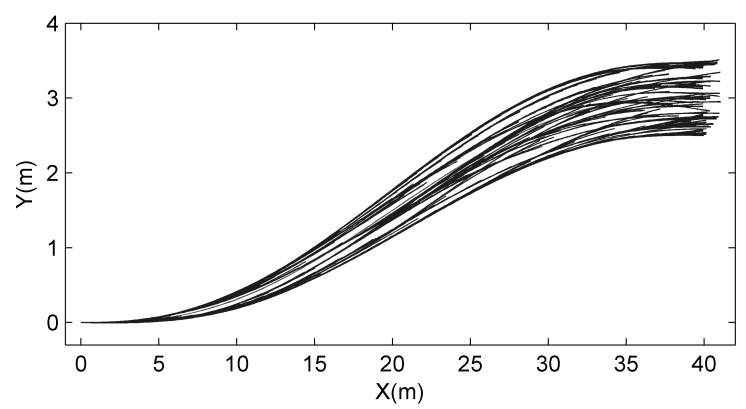

(b) Controller B

Fig. 8. RRT Analysis

\section{CONCLUSION}

The comparison of the two inversion-based controllers according to [24] and [7] showed that a set of test methodologies is required for a thorough evaluation: selected test cases for the most significant deviations in initial states and vehicle parameters, Monte-Carlo simulation for the average control performance, and rapidly exploring random trees for the worst-case performance.

The specific comparison of the inversion-based controllers only revealed significant differences with respect to the worst-case deviation, where the control concept proposed in [24] produced better results.

The test cases and test methodologies described in this work are fully specified, so that other researchers can use them as benchmark problems, or directly download the implementation from our website. We plan to add further test results of other control concepts on the website and encourage other researchers to send us their results. We also plan to test the most promising controllers on a real vehicle and compare the results with the simulations. 
TABLE III

RESULTS

\begin{tabular}{|c|c|c|c|c|c|c|c|c|}
\hline & \multicolumn{2}{|c|}{ max. deviation $(\mathrm{m})$} & \multicolumn{2}{|c|}{ avrg. deviation (m) } & \multicolumn{2}{|c|}{ final deviation $(\mathrm{m})$} & \multicolumn{2}{|c|}{ avrg. tire saturation } \\
\hline & $t$ & $n$ & $t$ & $n$ & $t$ & $n$ & $f$ & $r$ \\
\hline \multicolumn{9}{|c|}{ (i) Initial Deviation, Scenario I } \\
\hline A & $4.51 \cdot 10^{-3}$ & $4.42 \cdot 10^{-1}$ & $1.99 \cdot 10^{-3}$ & $2.21 \cdot 10^{-1}$ & $-9.44 \cdot 10^{-5}$ & $-1.56 \cdot 10^{-3}$ & 0.58 & 0.43 \\
\hline B & $1.60 \cdot 10^{-2}$ & $4.40 \cdot 10^{-1}$ & $7.19 \cdot 10^{-3}$ & $2.21 \cdot 10^{-1}$ & $-1.34 \cdot 10^{-3}$ & $1.21 \cdot 10^{-2}$ & 0.58 & 0.43 \\
\hline \multicolumn{9}{|c|}{ (i) Initial Deviation, Scenario II } \\
\hline A & $5.23 \cdot 10^{-3}$ & $4.51 \cdot 10^{-1}$ & $1.20 \cdot 10^{-3}$ & $1.16 \cdot 10^{-1}$ & $-7.61 \cdot 10^{-5}$ & $-4.47 \cdot 10^{-4}$ & 0.6 & 0.42 \\
\hline B & $1.47 \cdot 10^{-2}$ & $4.68 \cdot 10^{-1}$ & $4.12 \cdot 10^{-3}$ & $1.30 \cdot 10^{-1}$ & $4.80 \cdot 10^{-5}$ & $5.20 \cdot 10^{-3}$ & 0.63 & 0.4 \\
\hline \multicolumn{9}{|c|}{ (ii) Tire Force Saturation, $\mu_{0}=0.6$ known, Scenario I } \\
\hline A & $9.56 \cdot 10^{-3}$ & $1.21 \cdot 10^{-2}$ & $3.33 \cdot 10^{-3}$ & $4.01 \cdot 10^{-3}$ & $8.34 \cdot 10^{-3}$ & $1.16 \cdot 10^{-2}$ & 0.82 & 0.55 \\
\hline B & $1.22 \cdot 10^{-2}$ & $1.49 \cdot 10^{-2}$ & $3.59 \cdot 10^{-3}$ & $8.15 \cdot 10^{-3}$ & $1.10 \cdot 10^{-2}$ & $-6.89 \cdot 10^{-3}$ & 0.82 & 0.55 \\
\hline \multicolumn{9}{|c|}{ (ii) Tire Force Saturation, $\mu_{0}=0.6$ known, Scenario II } \\
\hline A & $1.56 \cdot 10^{0}$ & $1.01 \cdot 10^{0}$ & $5.33 \cdot 10^{-1}$ & $3.41 \cdot 10^{-1}$ & $1.45 \cdot 10^{0}$ & $-3.29 \cdot 10^{-1}$ & 0.96 & 0.54 \\
\hline B & $1.26 \cdot 10^{1}$ & $8.14 \cdot 10^{0}$ & $3.61 \cdot 10^{0}$ & $3.01 \cdot 10^{0}$ & $1.26 \cdot 10^{1}$ & $8.14 \cdot 10^{0}$ & 0.99 & 0.74 \\
\hline \multicolumn{9}{|c|}{ (ii) Tire Force Saturation, $\mu_{0}=0.6$ unknown, Scenario I } \\
\hline A & $1.97 \cdot 10^{-1}$ & $1.33 \cdot 10^{-1}$ & $8.88 \cdot 10^{-2}$ & $7.08 \cdot 10^{-2}$ & $1.84 \cdot 10^{-1}$ & $1.28 \cdot 10^{-1}$ & 0.83 & 0.5 \\
\hline B & $2.22 \cdot 10^{-1}$ & $8.96 \cdot 10^{-2}$ & $9.69 \cdot 10^{-2}$ & $4.32 \cdot 10^{-2}$ & $2.11 \cdot 10^{-1}$ & $6.72 \cdot 10^{-2}$ & 0.84 & 0.54 \\
\hline \multicolumn{9}{|c|}{ (ii) Tire Force Saturation, $\mu_{0}=0.6$ unknown, Scenario II } \\
\hline A & $1.89 \cdot 10^{0}$ & $1.41 \cdot 10^{0}$ & $6.69 \cdot 10^{-1}$ & $5.30 \cdot 10^{-1}$ & $1.81 \cdot 10^{0}$ & $-1.61 \cdot 10^{-1}$ & 0.93 & 0.49 \\
\hline B & $5.07 \cdot 10^{0}$ & $9.33 \cdot 10^{0}$ & $1.69 \cdot 10^{0}$ & $1.30 \cdot 10^{0}$ & $5.07 \cdot 10^{0}$ & $-9.33 \cdot 10^{0}$ & 0.93 & 0.83 \\
\hline \multicolumn{9}{|c|}{ (iii) Mismatched Parameters, Scenario I } \\
\hline A & $2.46 \cdot 10^{-1}$ & $6.73 \cdot 10^{-2}$ & $1.22 \cdot 10^{-1}$ & $3.95 \cdot 10^{-2}$ & $2.37 \cdot 10^{-1}$ & $6.59 \cdot 10^{-2}$ & 0.66 & 0.32 \\
\hline B & $2.47 \cdot 10^{-1}$ & $3.85 \cdot 10^{-2}$ & $1.22 \cdot 10^{-1}$ & $8.97 \cdot 10^{-3}$ & $2.38 \cdot 10^{-1}$ & $-3.85 \cdot 10^{-2}$ & 0.66 & 0.32 \\
\hline \multicolumn{9}{|c|}{ (iii) Mismatched Parameters, Scenario II } \\
\hline A & $1.82 \cdot 10^{-1}$ & $7.94 \cdot 10^{-2}$ & $1.10 \cdot 10^{-1}$ & $3.74 \cdot 10^{-2}$ & $1.07 \cdot 10^{-1}$ & $-7.94 \cdot 10^{-2}$ & 0.65 & 0.34 \\
\hline B & $1.83 \cdot 10^{-1}$ & $1.89 \cdot 10^{-1}$ & $1.10 \cdot 10^{-1}$ & $2.49 \cdot 10^{-2}$ & $1.04 \cdot 10^{-1}$ & $1.89 \cdot 10^{-1}$ & 0.66 & 0.35 \\
\hline
\end{tabular}

\section{REFERENCES}

[1] J. Andreasson and T. Bunte. Global chassis control based on inverse vehicle dynamics models. Vehicle System Dynamics, 44(sup1):321328, 2006.

[2] S. Antonov, A. Fehn, and A. Kugi. A new flatness-based control of lateral vehicle dynamics. Vehicle System Dynamics, 46(9):789-801, 2008.

[3] A. Benine-Neto, S. Scalzi, S. Mammar, and M. Netto. Dynamic controller for lane keeping and obstacle avoidance assistance system. In Intelligent Transportation Systems (ITSC), 13th International IEEE Conference on, pages 1363 -1368, 2010.

[4] A. Bhatia and E. Frazzoli. Incremental search methods for reachability analysis of continuous and hybrid systems. In Hybrid Systems: Computation and Control, LNCS 2993, pages 142-156. Springer, 2004.

[5] M. Buehler, K. Lagnemma, and S. Singh, editors. Special Issue on the 2007 DARPA Urban Challenge, Part 1-3, volume 25(8-10):423-860 of Journal of Field Robotics. Wiley, 2008.

[6] M. Fliess, J. Lévine, P. Martin, and P. Rouchon. Flatness and defect of non-linear systems: introductory theory and examples. International Journal of Control, 61(6):1327-1361, 1995.

[7] S. Fuchshumer, K. Schlacher, and T. Rittenschober. Nonlinear vehicle dynamics control - a flatness based approach. In Decision and Control, European Control Conference. CDC-ECC'05. 44th IEEE Conference on, pages 6492-6497, 2005.

[8] K. Graichen, V. Hagenmeyer, and M. Zeitz. A new approach to inversion-based feedforward control design for nonlinear systems. Automatica, 41(12):2033-2041, 2005.

[9] A. Isidori. Nonlinear control systems, volume 1. Springer Verlag, 1995.

[10] S. M. LaValle. Planning Algorithms. Cambridge University Press, 2006.

[11] S. M. LaValle and J. J. Kuffner. Randomized kinodynamic planning. International Journal of Robotics Research, 20(5):378-400, 2001.

[12] R. Mayr. Verfahren zur Bahnfolgeregelung für ein automatisch geführtes Fahrzeug. PhD thesis, Universität Dortmund, 1991.

[13] J. Minguez, L. Montana, and O. Khatib. Reactive collision avoidance for navigation with dynamic constraints. In Proc. of the IEEE International Conference on Intelligent Robots and Systems, pages 588-594, 2002.
[14] R. Orend. Integrierte Fahrdynamikregelung mit Einzelradaktorik - Ein Konzept zur Darstellung des fahrdynamischen Optimums. PhD thesis, Universität Erlangen-Nürnberg, 2006.

[15] H. B. Pacejka. Tyre and Vehicle Dynamics. Butterworth Heinemann, 2005.

[16] S. Peters. Optimal Planning and Control for Hazard Avoidance of Front-Wheel Steered Ground Vehicles. PhD thesis, Massachusetts Institute of Technology, 2012.

[17] S. Peters, E. Frazzoli, and K. Iagnemma. Differential flatness of a front-steered vehicle with tire force control. In Intelligent Robots and Systems (IROS), 2011 IEEE/RSJ International Conference on, pages $298-304$, sept. 2011.

[18] D. Ren, J. Zhang, J. Zhang, and S. Cui. Trajectory planning and yaw rate tracking control for lane changing of intelligent vehicle on curved road. Science China Technological Sciences, 54:630-642, 2011.

[19] E. J. Rossetter and J. C. Gerdes. Lyapunov based performance guarantees for the potential field lane-keeping assistance system. Journal of Dynamic Systems, Measurement and Control, 128(3):510523, 2006.

[20] R. Rothfuß, J. Rudolph, and M. Zeitz. Flachheit: Ein neuer Zugang zur Steuerung und Regelung nichtlinearer Systeme. at - Automatisierungstechnik, 45:517-525, 1997.

[21] P. Setlur, J. Wagner, D. Dawson, and D. Braganza. A trajectory tracking steer-by-wire control system for ground vehicles. Vehicular Technology, IEEE Transactions on, 55(1):76-85, 2006.

[22] E. Velenis, E. Frazzoli, and P. Tsiotras. On steady-state cornering equilibria for wheeled vehicles with drift. In Decision and Control,Proc. of the 48th IEEE Conference on, pages 3545-3550, 2009.

[23] J. Villagra, B. d'Andrea Novel, H. Mounier, and M. Pengov. Flatnessbased vehicle steering control strategy with SDRE feedback gains tuned via a sensitivity approach. Control Systems Technology, IEEE Transactions on, 15(3):554-565, 2007.

[24] M. Werling. Ein neues Konzept für die Trajektoriengenerierung und -stabilisierung in zeitkritischen Verkehrsszenarien. $\mathrm{PhD}$ thesis, Karlsruher Institut für Technologie, 2010. 